\title{
E-Sports: a legitimate sport?
}

Currently, our society is facing an increase in the levels of physical inactivity in the world population, especially an increase in screen time, causing potential adverse effects in the short and long term to health (Stiglic \& Viner, 2019; Domingues-Montanari, 2017). Several institutions, such as the World Health Organization (WHO), American College of Sports Medicine (ACSM), and National Institute for Health and Care Excellence (NICE), recognize the importance of physical exercise as a potential tool against the risks associated with sedentary behavior (Piercy et al., 2018). And within that context, a question must be asked; can screen time be considered time spent on sports?

In the meantime, sports can be defined as activities involving physical effort, motor skills as the main focus of the activity, with elements of competition where rules and behavior patterns that govern the activity formally exist through organizations (Budde et al., 2016). Therefore, sports practice can include any type of physical activity or physical exercise and is influenced by different contexts and individual disposition. On the other hand, there is still no consensual definition for electronic sports (e-Sports). Generally, e-Sports include several different types of electronic games, played competitively in controlled environments, with structure and regulations similar to traditional sports (for example, leagues and tournaments) (Hallmann \& Giel, 2018; Karhulahti, 2017), and with different categories, such as sports, with FIFA as one of the main games in the category.

In the last decade, e-Sports have grown exponentially, reaching a high level of competitiveness with traditional sports. Thousands of players play daily games and electronic competitions, promoting a fast proliferation around the world. Several professional e-Sports competitions have emerged for a similar structure, achieving great popularity (social media and the public) and generating a lot of revenue, as well as several other professional sports (Jenny et al., 2017), despite they have received a lot of criticism due to their lack of physicality when compared to traditional sports. E-Sports players differ from casual electronic game players, they are professional players who play for competition, not for fun and/or relaxation (Witkowski, 2012). E-Sports as an area of sports activities in which people develop and train physical and mental skils in the use of information and communication technologies (Hallmann \& Giel, 2018), in addition to being considered alternative sports (Adamus 2012). Therefore, another important question must also be asked; would E-Sports athletes be legitimate athletes?

In an attempt to answer this question, we can use exergames as an example, which is the combination of electronic games and physical exercise, since they use movements as an interface with the game itself, thus acting in the maintenance and improvement of physical capacities (Bamparopoulos et al., 2016). An interesting study conducted by Tuomas and VeliMatti (2016), showed that e-Sports athletes spend about 5.3 hours per day in training, including 1.1 hours of physical exercise. However, the time spent by these athletes in sedentary activities is excessive, and this is a worrying factor due to the development of several diseases. In this study, of 115 e-Sports athletes, $64.3 \%(n=74)$ practiced physical exercise for more than 1 hour a day, meeting the recommendations of WHO, ACSM and NICE. And of the total of athletes, more than half $(55.6 \%, \mathrm{n}=64)$ believed that the practice of exercises can affect their performance. Therefore, this concept is a good catalyst for promoting physical activity among e-Sports athletes.

In line with Tuomas and Veli-Matti (2016), other study showed that e-Sports athletes have high levels of physical activity (Pereira et al., 2019). The authors investigated 928 e-Sports athletes 
registered with the FPF, indicating that 721 had some level of physical activity, $73 \%$ of which were classified as having high levels of physical activity. According to the recommendations of WHO, ACSM and NICE, athletes reached from minimum levels (ie, 1500 MET-minutes/week or 3 days a week) to high levels of physical activity (ie, 3000 MET-minutes/week or $\geq 7$ days a week). Corroborating the findings of Pereira et al. (2019), a Finnish study revealed that professional Finnish e-Sports athletes have physical activity levels three times higher than the recommendations of WHO, ACSM and NICE. However, as much as these athletes have high levels of physical activity, what about the general adverse effects on the physical and psychological health of a high amount of screen time due to e-Sports training and competitions? (DiFrancisco-Donoghue et al., 2019).

In this sense, very little is known about the psychological aspects and physiological demands of e-Sports competitions, as well as their psychological and physical health in the short and long term. It is well known that elevated screen time is associated with deleterious effects on sleep, psychological status, and cognitive functions, as well as repetitive strain injuries. With regard to physical health, e-Sports athletes perform movements similar to other occupations that require long periods using computers, repetitive or forced movements, static postures, poorly lit environments and sitting in the same position for a long time, such as, administrative and technology professionals (European Agency for Safety and Health at Work, 2018). These factors are critical for the development of musculoskeletal injuries. Corroborating the findings above, a recent study analyzed 65 university athletes from the USA and Canada in e-Sports and found that athletes were susceptible to injuries from overuse, with neck and back pain being the most prevalent (DiFrancisco-Donoghue et al., 2019).

As for the psychological state, with the increase in popularity in the media and the social impact of e-Sports, the greater the number of competitions, awards or sponsorships, and consequently, the pressure around positive results, demands commonly faced by any professional athlete. (Witkowski, 2012; Karhulahti, 2017). An extremely important point is that a high level of pressure suffered can predispose these e-Sports athletes to be affected by psychiatric disorders. In the latest edition of the International Classification of Diseases, the 11th (ICD-11), "gambling disorder" was included, as one of the disorders related to addictive behaviors (World Health Organization, 2019). Within this context, e-Sports can be a trigger factor for gambling disorder, although evidence suggests that the practice of electronic games affects only a small percentage of practitioners (World Health Organization, 2018).

Although professional e-Sports athletes are a small number when compared to the thousands of regular e-Sports practitioners (Brock, 2017), there is a great need to better understand the environment and the potential risks arising from "work", to better anticipate and prevent possible health problems. Therefore, due to the increasing increase in competitiveness and professionalization and, also, the little knowledge about the health of e-Sports athletes, more knowledge and investments in research in the area are needed. Support programs for e-Sports players must be designed and implemented, as well as health guidelines or recommendations must be written and promoted.

\section{References}

1. Adamus, T. (2012). Playing computer games as electronic sport: In search of a theoretical framework for a new research field. In Computer games and new media cultures (pp. 477-490). Springer, Dordrecht.

2. Bamparopoulos, G., Konstantinidis, E., Bratsas, C., \& Bamidis, P. D. (2016). Towards exergaming commons: composing the exergame ontology for publishing open game data. Journal of Biomedical Semantics, 7(1), 4. 
Brock, T. (2017). Roger Caillois and e-sports: On the problems of treating play as work. Games and Culture, 12(4), 321-339.

Budde, H., Schwarzc, R., Velasques, B., Ribeiro, P., Holzweg, M., Machado, S., ... \& Wegner, M. (2016). The need for differentiating between exercise, physical activity, and training. Autoimmunity Reviews, 15(1), 110-1.

DiFrancisco-Donoghue, J., Balentine, J., Schmidt, G., \& Zwibel, H. (2019). Managing the health of the eSport athlete: an integrated health management model. BMJ open Sport \& Exercise Medicine, 5(1), e000467.

Domingues-Montanari, S. (2017). Clinical and psychological effects of excessive screen time on children. Journal of Paediatrics and Child Health, 53(4), 333-338.

European Agency for Safety and Health at Work. Musculoskeletal disorders (2018). Available: https://osha.europa.eu/en/themes/musculoskeleta 1- disorders [Accessed May 2019].

Hallmann, K., \& Giel, T. (2018). eSportsCompetitive sports or recreational activity?. Sport Management Review, 21(1), 14-20.

Jenny, S. E., Manning, R. D., Keiper, M. C., \& Olrich, T. W. (2017). Virtual (ly) athletes: where eSports fit within the definition of "Sport". Quest, 69(1), 1-18.

Karhulahti, V. M. (2017). Reconsidering esport: Economics and executive ownership. Physical
Culture and Sport. Studies and Research, 74(1), 43-53.

Pereira, A. M., Figueiredo, P., Seabra, A., \& Brito, J. (2019). Evaluation of physical activity levels in FPF eSports e-athletes. Motricidade, 15, 188-188.

Piercy, K. L., Troiano, R. P., Ballard, R. M., Carlson, S. A., Fulton, J. E., Galuska, D. A., ... \& Olson, R. D. (2018). The physical activity guidelines for Americans. Jama, 320(19), 20202028.

Stiglic, N., \& Viner, R. M. (2019). Effects of screentime on the health and well-being of children and adolescents: a systematic review of reviews. BMJ Open, 9(1), e023191.

Tuomas K, Veli-Matti K. (2016). Do e-athletes move? A study on training and physical exercise in elite e-sports. Intertational Journal Gaming Computer-Mediated Simulations, 8(4), 53-66.

Witkowski, E. (2012). On the digital playing field: How we "do sport" with networked computer games. Games and Culture, 7(5), 349374.

World Health Organization. Gaming disorder (2018). Available: https://www. who.int/features/qa/gaming-disorder/en/

World Health Organization. ICD-11 for mortality and morbidity statistics: 6C51 gaming disorder. version: $\quad 04, \quad 2019 . \quad$ Available: http://id.who.int/icd/entity/14485972

Matheus Cerqueira1,4, Diogo Monteiro2, Bruno Travassos3, Sergio Machado1,3,4

1Laboratory of Physical Activity Neuroscience, Physical Activity Sciences Post-Graduate Program (PGCAF), Salgado de Oliveira University, Niterói, Brazil. ${ }_{2}$ Sport Science School of Rio Maior, ESDRM-IP Santarém, Rio Maior, Portugal; Research Center in Sports Sciences, Health Sciences and Human Development (CIDESD), Vila Real, Portugal. 3Department of Sports Science, University of Beira Interior, 6201-001, Covilhã, Portugal; Research Centre in Sports, Health and Human Development (CIDESD), 6201-001, Covilhã, Portugal; Portugal Football School da FPF, Lisboa, Portugal. ${ }_{4}$ Comissão Técnica da Equipe de e-Sports Black Dragons, São Paulo, Brasil 